\title{
Exponentes de escala de las funciones de estructura longitudinales y transversales en turbulencia homogénea e isotrópica generada en un túnel de viento Scale exponents of longitudinal and transverse structure functions in homogeneous and isotropic turbulence generated in a wind tunnel
}

\author{
Laura Velásquez García ${ }^{1,2}$ \\ ${ }^{1}$ Departamento de Ingeniería Mecánica, Facultad de Ingeniería, Universidad EAFIT, Medellín, Colombia. \\ Correo electrónico: 1velas30@eafit.edu.co \\ ${ }^{2}$ Grupo Energía Alternativa, Departamento de Ingeniería Mecánica, Facultad de Ingeniería, Universidad de \\ Antioquia, Medellín, Colombia. Correo electrónico: lisabel.velasquez@udea.edu.co
}

Recibido: 29 junio, 2018. Aceptado: 28 diciembre, 2018. Versión final: 6 febrero, 2019.

\begin{abstract}
Resumen
Usando una malla activa y una malla pasiva se caracterizó un flujo turbulento homogéneo e isotrópico estudiando los exponentes de las funciones de estructura longitudinal $\left\langle\Delta \mathrm{u}(\mathrm{x})^{\mathrm{n}}\right\rangle$ y transversales $\left.\left\langle\Delta \mathrm{u}(\mathrm{y})^{\mathrm{n}}\right\rangle\right\rangle$ y $\left\langle\Delta \mathrm{v}(\mathrm{x})^{\mathrm{n}}\right\rangle$, donde $\mathbf{u}$ es la fluctuación de la velocidad longitudinal, y $\mathbf{v}$ es la fluctuación de la velocidad transversal, en función del número de Reynolds $\mathrm{R}_{\lambda}$ para órdenes, $\mathrm{n}$, entre 2 y 8 . La turbulencia se generó en un túnel de viento horizontal de $91 \times 91 \mathrm{~cm}^{2} \mathrm{de}$ sección transversal, y 9,14 m de longitud. El campo de velocidades se midió con anemometría de hilos calientes con sensores TSI 1241 y TSI 1210 fabricados con una aleación de platino y rodio de 2,54 $\mu \mathrm{m}$ de diámetro. Los resultados indicaron que los exponentes de escala varían en función de $R_{\lambda}$, cuanto más aumentaba $R_{\lambda}$, mayor era la diferencia entre los exponentes de las funciones de estructura y los exponentes predichos por la teoría de Kolmogorov sobre turbulencia.
\end{abstract}

Palabras clave: anemometría de hilos calientes; funciones de estructura; malla activa; malla pasiva; túnel de viento; turbulencia homogénea; turbulencia isotrópica.

\begin{abstract}
By using an active grid and a passive grid, a homogenous and isotropic turbulent flow was characterized by studying its longitudinal structure functions $\left\langle\Delta \mathrm{u}(\mathrm{x})^{\mathrm{n}}\right\rangle$ and transverse $\left.\left\langle\Delta \mathrm{u}(\mathrm{y})^{\mathrm{n}}\right\rangle\right\rangle$ and $\left\langle\Delta \mathrm{v}(\mathrm{x})^{\mathrm{n}}\right\rangle$ where $\mathbf{u}$ is the longitudinal velocity fluctuation, and $\mathbf{v}$ is the fluctuation of the transverse velocity, as a function of the Reynolds number $R_{\lambda}$ for orders, $n$, between 2 and 8 . The turbulence was generated in a wind tunnel with $91 \times 91 \mathrm{~cm}^{2}$ in cross-section and $9,14 \mathrm{~m}$ in length. The velocity field was measured with hot-wire anemometry with TSI 1241 and TSI 1210 sensors manufactured with an alloy of platinum and rhodium of 2,54 $\mu \mathrm{m}$ diameter. The results indicated that the exponents of scale vary according to $\mathrm{R}_{\lambda}$; if $\mathrm{R}_{\lambda}$ increases, the difference between the exponents of the structure functions and the exponents predicted by the $\mathrm{K} 41$ theory also increases.
\end{abstract}

ISSN impreso: 1657 - 4583. ISSN en línea: 2145 - 8456, CC BY-ND 4.0 (c) (i)

L. Velásquez, "Exponentes de escala de las funciones de estructura longitudinales y transversales en turbulencia homogénea e isotrópica generada en un túnel de viento," Rev. UIS Ing., vol. 18, no. 2, pp. 89-102, 2019. doi: 10.18273/revuin.v18n2-2019008 
Keywords: active grid; homogenous turbulence; isotropic turbulence; structure function; passive grid; hot-wire anemometry; wind tunnel.

\section{Introducción}

La turbulencia generada en túneles de viento a través del paso de un flujo de aire por mallas ha sido extensamente estudiada por más de 50 años. Estos túneles son comúnmente usados para crear turbulencia homogénea e isotrópica, gracias a que es posible controlar las condiciones de operación. La turbulencia isotrópica es el tipo de turbulencia donde el valor de cualquier función de las componentes de la velocidad y sus derivadas en un punto, definido en relación a un conjunto particular de ejes, son inalterados si los ejes de referencia son rotados en cualquier dirección, o si el sistema coordenado es reflejado en cualquier plano a través del origen. La turbulencia homogénea describe un tipo de turbulencia cuyas propiedades estadísticas son independientes de la posición en el fluido. Por tanto, la turbulencia isotrópica y homogénea representa la turbulencia, que es la misma en todas las direcciones y en todas las posiciones.

Entre los diferentes análisis que pueden realizarse sobre este fenómeno, las propiedades estadísticas de la turbulencia homogénea e isotrópica a menudo se investigan mediante el estudio de las funciones de estructura. Las funciones de estructura de orden $n$ para la velocidad longitudinal u se definen así:

$$
\left\langle\Delta u(x)^{n}\right\rangle=\left\langle(u(x+r)-u(x))^{n}\right\rangle
$$

Donde $u(x+r)$ y $u(x)$ son velocidades a lo largo del eje $\mathbf{x}$, eje paralelo al flujo, para dos puntos separados por una escala o distancia, $\mathrm{r}, \mathrm{y}\langle-\rangle$ indica valores promedio.

Para la turbulencia homogénea e isotrópica, Kolmogorov estableció en su teoría K41 [1] que la relación entre las funciones de estructura y $r$, que corresponde a una distancia dentro del rango inercial, podía representarse siguiendo una ley de potencias:

$$
\left\langle\Delta \boldsymbol{u}(\boldsymbol{x})^{n}\right\rangle \sim \boldsymbol{r}^{\xi_{u(x)}^{n}}
$$

Donde $\xi_{u(x)}^{n}$ es el exponente de escala de la función de estructura longitudinal $\Delta u(x)^{n}$ de orden n. El rango inercial corresponde a longitudes donde la viscosidad y la tasa de difusividad son despreciables, $L>>r>>\eta$, donde es $\eta$ la escala disipativa, y $L$ es la escala integral (escala donde la energía es inyectada). Kolmogorov supuso además que, como la tasa de disipación, $\epsilon$, permanecía constante en este rango, existía una dependencia lineal entre los exponentes de escala y el orden de la función de estructura, $\xi_{u(x)}^{n}=n / 3$. Sin embargo, existe una diferencia significativa entre su predicción y los resultados experimentales. Estas diferencias o desviaciones respecto de esa forma lineal se conocen como intermitencia de la turbulencia a pequeña escala.

Es posible también, con la descripción de Kolmogorov, el cálculo de las funciones de estructura transversal, de la que existen dos tipos: la función de estructura para la velocidad transversal, $v$, medida en la dirección del flujo, $\mathrm{x}$, que se calcula mediante la ecuación $\left\langle\Delta \mathrm{v}(\mathrm{x})^{\mathrm{n}}\right\rangle=$ $\left\langle(\mathrm{v}(\mathrm{x}+\mathrm{r})-\mathrm{v}(\mathrm{x}))^{\mathrm{n}}\right\rangle$, y habrá una serie de valores diferentes para sus exponentes de escala, $\xi_{v(x)}^{n}$; y la función de estructura para la velocidad longitudinal, u, medida con separaciones en dirección transversal al flujo, $\mathrm{y}$, que se calcula mediante la ecuación $\left\langle\Delta \mathrm{u}(\mathrm{y})^{\mathrm{n}}\right\rangle=$ $\left\langle(\mathrm{u}(\mathrm{y}+\mathrm{r})-\mathrm{u}(\mathrm{y}))^{\mathrm{n}}\right\rangle$, y que también tendrá una serie de valores propios para los exponentes de escala, $\xi_{u(y)}^{n}$.

En este reporte se documentan los resultados de una investigación experimental de flujos turbulentos. Estos experimentos estuvieron enfocados en la medición de los exponentes de escala longitudinal y transversal para turbulencia generada en un túnel de viento por una malla pasiva y una malla activa. Un aspecto importante de estos experimentos fue la posibilidad de medir simultáneamente las funciones de estructura $\left\langle\Delta \mathrm{u}(\mathrm{x})^{\mathrm{n}}\right\rangle$ y $\left\langle\Delta \mathrm{v}(\mathrm{x})^{\mathrm{n}}\right\rangle$ usando sensores bidimensionales.

\section{Instalación experimental}

La turbulencia fue generada en un túnel de viento horizontal, de succión y de circuito abierto de $91 \times 91 \mathrm{~cm}^{2}$ de sección transversal y 9,14 m de longitud ubicado en la Facultad de Ingeniería Mecánica y Aeroespacial de la Universidad de Cornell, en Ithaca, Estados Unidos. Un túnel de viento de succión y de circuito abierto es aquel en que el aire se aspira directamente de la atmósfera, y después de hacerlo pasar por la sección de ensayo o prueba, se devuelve nuevamente a ella. El túnel fue diseñado inicialmente para el estudio de flujos estratificados que involucraban no solo perfiles de velocidad, sino también de temperatura [4]. Un esquema del túnel con los diferentes elementos que lo componen se proporciona en la figura 1 .

El túnel fue fabricado con madera contrachapada de 0,19 $\mathrm{m}$ de espesor, $\mathrm{y}$ estaba cubierto con hojas aislantes de fibra de vidrio de $0,05 \mathrm{~m}$ de espesor. La sección de ensayo permitía el acceso óptico hacia el interior del 
túnel, a través de 34 agujeros ubicados en las paredes laterales y 18 agujeros en las paredes superior e inferior. Una de las paredes laterales del túnel era ligeramente divergente, con ángulo de paso de $0,3^{\circ}$, para asegurar una velocidad media constante en la línea central del túnel a pesar del desarrollo de las capas límite; la superficie

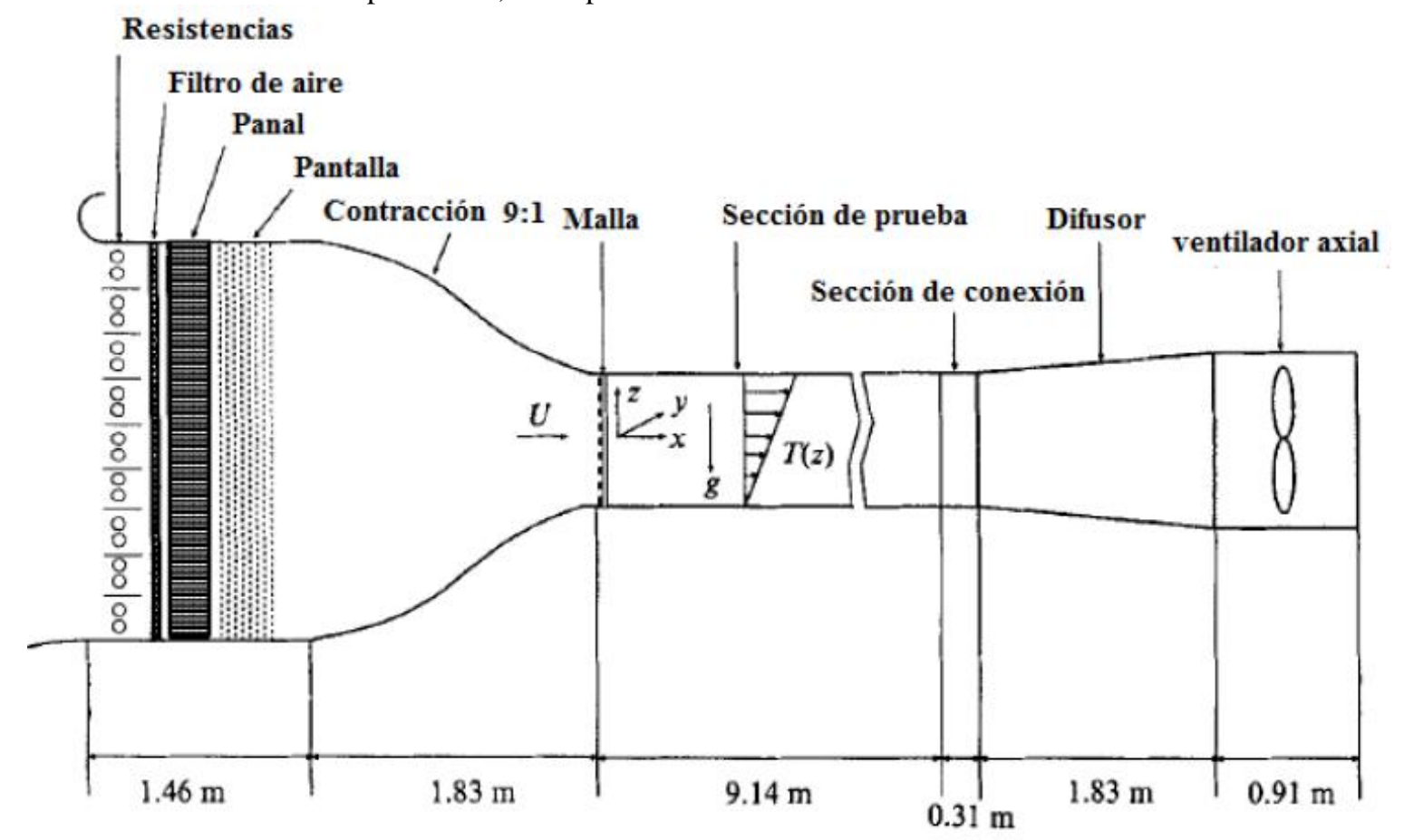

interna de la sección de pruebas no tenía una rugosidad significativa, para que las variaciones de velocidad y presión dinámica del flujo no superaran el $25 \%$ y $50 \%$ de sus valores medios, respectivamente.

Figura 1: Esquema del Túnel de viento. Fuente: Kyunghwan Yoon and Z Warhaft, 1990.

El flujo fue acelerado antes de entrar en la sección de ensayo en una contracción con una relación de áreas de 9:1 de 1,83 m longitud. En la unión entre la contracción y la sección de ensayo fue instalada la malla encargada de la generación del flujo turbulento. El final de la sección de ensayo se conectó a un difusor, en el que se hacía una transición suave de la sección cuadrada a una sección circular que albergaba un ventilador axial 1,24 m de diámetro. El ventilador era alimentado por un motor de $60 \mathrm{HP}$, que giraba a una velocidad máxima de 1070 rpm. El motor que impulsaba este ventilador estaba situado en el exterior del túnel de viento, aislado del resto del túnel para reducir la transmisión de vibraciones mecánicas. El motor del ventilador estaba conectado a un variador de frecuencia que permitía ajustar la velocidad de giro del motor con incrementos de $0,5 \mathrm{~Hz}$, para generar diferentes velocidades dentro del túnel. La velocidad máxima resultante de la instalación fue de 20 $\mathrm{m} / \mathrm{s}$. Para las pruebas se seleccionaron velocidad $U_{0}$ del aire dentro del túnel de 3, 6, 9 y $12 \mathrm{~m} / \mathrm{s}$, lo que generó variaciones para el número de Reynolds definido en función de la escala de Taylor $\lambda$ de 100 hasta 1200 .
Los generadores de turbulencia pasivos son el método más simple para producir turbulencia aguas abajo en túneles de viento. Una malla pasiva consiste en un arreglo de barras cilíndricas o planas que forman una sección cuadrada. La malla pasiva usada en las mediciones estaba compuesta por 8 barras planas horizontales y 8 barras planas verticales de aluminio, con un espesor de $19 \mathrm{~mm}$ unidas a un marco, figura 2a. Las barras están separadas por una distancia de $8,26 \mathrm{~cm}$, horizontal y verticalmente, lo que produce una separación entre los puntos medios de cada barra, M, de 10,16 $\mathrm{cm}$. Una malla activa es un generador de turbulencia que utiliza elementos activos para producir turbulencia. La malla activa estilo Makita [2] es un conjunto de 100 alas cuadradas acopladas a una malla de barras redondas. Las barras cilíndricas son accionadas por motores paso a paso (200 pasos por revolución) en una secuencia aleatoria para crear una distribución de obstrucción transitoria en la sección de entrada de prueba del túnel de viento. La malla activa está compuesta por 8 barras horizontales y 7 barras verticales de aluminio con un diámetro de $12,7 \mathrm{~mm}$ unidas a un marco a través de rodamientos de baja fricción, figura $2 b$. 
Las barras están separadas horizontal y verticalmente por una distancia de $82,6 \mathrm{~mm}$, lo que produce una separación M de 12,1 cm. Ambas mallas fueron diseñadas $y$ fabricadas para experimentos previos realizados en el mismo túnel de viento [3][4] [5].

El campo de velocidades fue medido con anemometría de hilos calientes en 17 diferentes posiciones, o puntos de monitoreo, a lo largo de túnel usando anemómetros de temperatura constante Dantec 55M01. Un anemómetro de hilo caliente consiste en un filamento calentado que queda expuesto al paso de un flujo. Este filamento está conectado a un circuito eléctrico que es capaz de monitorear las variaciones de resistencia eléctrica por la acción del flujo. Entonces, se puede establecer una relación de pertinencia entre la velocidad del flujo y la resistencia observada en el filamento calentado a determinada temperatura.

Para la adquisición de datos fueron usados dos tipos de hilos calientes: sensor unidimensional TSI 1210 y sensor bidimensional TSI 1241. El sensor TSI 1241, figura 3, fue usado para medir las componentes axial y tangencial de la velocidad, componentes necesarios para el cálculo de las funciones de estructuras $\left\langle\Delta \mathrm{u}(\mathrm{x})^{\mathrm{n}}\right\rangle$ y $\left\langle\Delta v(x)^{n}\right\rangle$. Para la medición de $\left\langle\Delta u(y)^{n}\right\rangle$ fueron usados dos sensores TSI 1210, uno de los cuales estaba ubicado en una posición fija dentro del túnel, mientras que el otro se movía de forma perpendicular al flujo, para que la función de estructura en dirección $\mathbf{y}$ pudiera ser determinada.

Todos los sensores fueron fabricados de manera manual, con hilos de aleación de platino y rodio (al $10 \%$ ) de 2,54 $\mu \mathrm{m}$ de diámetro. Los hilos fueron calibrados usando el enfoque de ángulo efectivo, antes y después de cada medición, para encontrar las constantes de la ley de King [7], que luego permitirían relacionar la velocidad del flujo con el voltaje necesario para mantener el hilo a la temperatura fijada dentro del flujo.

Para la adquisición de los datos se utilizó una tarjeta de adquisición de datos NI USB-6221 de ocho canales; por cada velocidad y cada localización medida dentro del túnel el tamaño de la muestra fue de 1638400 datos; la adquisición de la señal fue controlada con el software comercial LabView. Debido al elevado volumen de datos experimentales recogidos, se procedió a su reducción mediante técnicas estadísticas para ser analizada en el dominio del tiempo y de la frecuencia, este proceso de reducción condujo a obtener en cada punto de medición un solo valor característico por cada variable de interés; el posprocesamiento de los datos fue llevado a cabo con el software comercial Matlab.
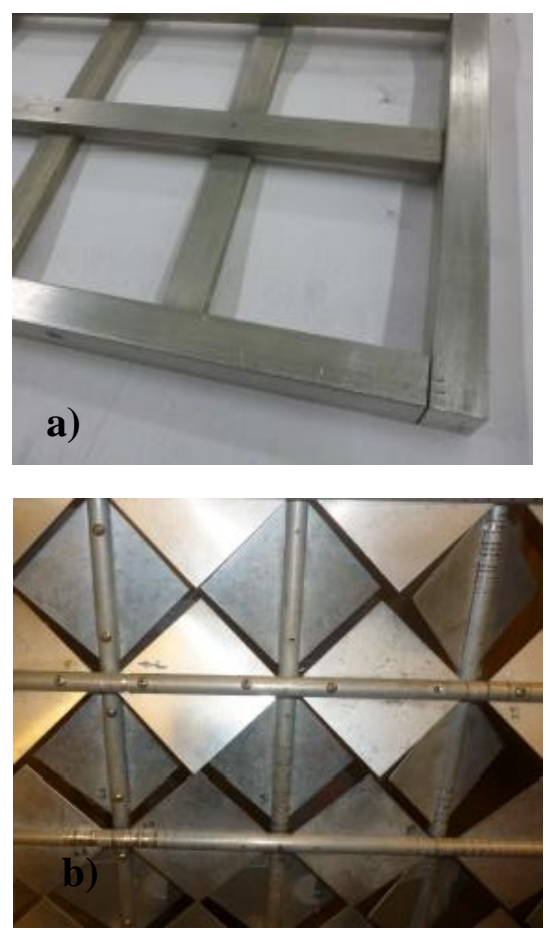

Figura 2. (a) Malla pasiva y (b) Malla activa. Fuente: elaboración propia.

\section{Resultados}

\subsection{Homogeneidad e isotropía}

Hay varias características clave que deben buscarse para verificar que la turbulencia producida por una malla sea homogénea e isotrópica. Por lo general, la velocidad media es homogénea dentro de un umbral de $\pm 1 \%$ [7]. En segundo lugar, la relación de isotropía, que es la relación de las raíces medias cuadráticas de la velocidad longitudinal y transversal, deberá ser la unidad, en un caso ideal, aunque normalmente toma valores entre 1,2 y 1,4 [8]. Además, la distribución estadística de la turbulencia debe ser aproximadamente Gaussiana, y la gráfica de la distribución de probabilidad debería colapsar para diferentes posiciones en el flujo [2] [7] [9] [10].

La homogeneidad e isotropía del campo de velocidad fue analizada tomando mediciones en dirección transversal al flujo en diferentes posiciones x para ambas mallas. $\mathrm{La}$ distancia x corresponde a la separación entre el sensor de medición y la malla; por tanto, una distancia $x=0$ corresponde a la ubicación de la malla. Durante la primera parte del estudio, se usó la malla pasiva y se midieron velocidades $U_{0}$ de 3,6 y $12 \mathrm{~m} / \mathrm{s}$, se tomaron mediciones para distancias x entre $482 \mathrm{~mm}$ y $6070 \mathrm{~mm}$; para la segunda parte, se usó la malla activa, con 
velocidades $U_{0}$ de 3,6 y $9 \mathrm{~m} / \mathrm{s}$ para distancias x entre $3530 \mathrm{~mm}$ y $7086 \mathrm{~mm}$. Para la malla activa no se midieron posiciones cercanas a la malla. $U_{0}$ fue medida con un tubo Pitot al final del túnel.

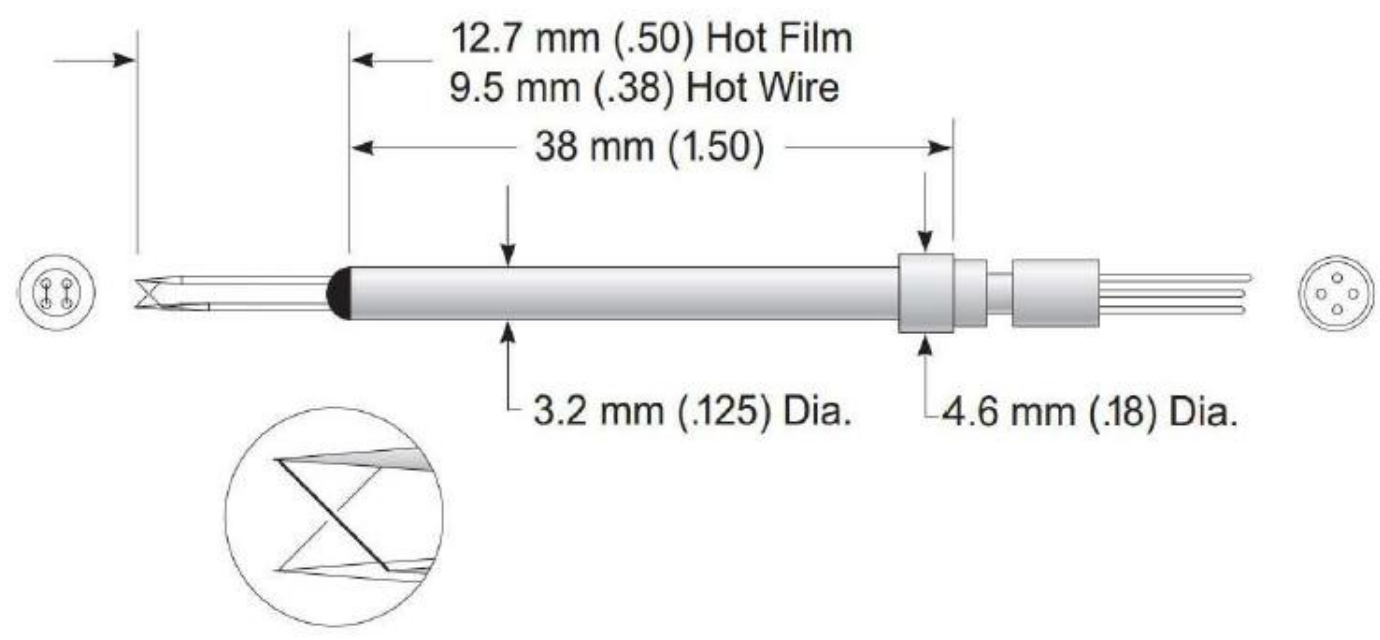

Figura 3. Esquema del sensor TSI 1241 con sus medidas características. Fuente: DANTEC DYNAMICS, 2012.

La figura 4 muestra las variaciones transversales de la velocidad media $\langle U\rangle$ normalizada por la velocidad del viento $U_{0}$, como función de la posición transversal y para diferentes posiciones x para malla pasiva y activa, para solo una velocidad de operación por cada malla. Las distancias $\mathbf{x}$ y $\mathbf{y}$ fueron normalizadas por el tamaño de malla M. La coordenada $y=0$ corresponde al centro del túnel. La sección de prueba para la malla pasiva comenzó en $x / M=4,75$, mientras que para la malla activa la sección inició en $x / M=29,18$.

La figura 4 indica que los perfiles transversales de la velocidad media normalizada en la malla pasiva son no homogéneos cerca a la malla, pero esta falta de homogeneidad disminuyó con el aumento de $\mathrm{x}$, debido a la acción de la difusividad, consistente con los resultados presentados por [11]. Las variaciones más pronunciadas de $\langle\mathrm{U}\rangle / U_{0}$, que implican mayor falta de homogeneidad, se generaron en la malla pasiva para una velocidad $U_{0}=6$ $\mathrm{m} / \mathrm{s}$, en donde la relación $\langle\mathrm{U}\rangle / U_{0}$ tomó valores de $1,32 \mathrm{en}$ $x / M=4.75$. Esta falta de homogeneidad cerca a la malla es causada por la formación de estelas al paso del aire, a través de las barras que conforman la malla.

Para las mediciones con malla activa no hubo problemas de homogeneidad en los perfiles transversales de la velocidad media, porque las mediciones se realizaron a mayores distancias de la malla. La relación $\langle\mathrm{U}\rangle / U_{0}$ fue muy similar en las cinco posiciones $x / M$ medidas en cada velocidad. Para la posición $x / M=45,97$ esta relación tendió a 1,26, 1,08 y 1,01 para las velocidades $U_{0}$ de 3,6 y $9 \mathrm{~m} / \mathrm{s}$, respectivamente, lo que indicó que cuanto mayor fue $U_{0}$ más cercano fue $\langle\mathrm{U}\rangle / U_{0}$, a la unidad, que es el valor esperado para la turbulencia homogénea. Para la malla activa se puede asegurar que por los valores de $\langle\mathrm{U}\rangle / U_{0}$ se está siempre ante un flujo homogéneo, mientras que para la malla pasiva se requiere que el valor de $x / M$ sea mayor de 19,75 .

Para evaluar la distancia desde la malla donde el flujo es isotrópico, se introduce el factor de isotropía, que es la relación de las raíces medias cuadráticas de las fluctuaciones de la velocidad longitudinal y transversal, $u^{\prime} / v^{\prime}$, donde $u^{\prime}=\sqrt{\left\langle u^{2}\right\rangle} y v^{\prime}=\sqrt{\left\langle v^{2}\right\rangle}$; el factor de isotropía deberá ser 1,0 cuando el flujo es isotrópico. En experimentos previos con mallas activas se reportaron valores de $u^{\prime} / v^{\prime}$ cercanos a 1,21 [5] y para mallas pasivas valores de 1,1 o ligeramente menores [8]. Valente y Vassilicos [12] han obtenido en sus experimentos con mallas pasivas valores de anisotropía en el rango de 1,10 y 1,15 .

La figura 5 muestra el factor de isotropía como función de la posición transversal $\boldsymbol{y}$ para ambos tipos de malla. El factor de isotropía fue de aproximadamente 1,05 para la malla pasiva y de 1,11 para la malla activa, que son valores muy cercanos a los valores de referencia. Este factor disminuyó al aumentar $U_{0}$ y fue constante en todo el rango de medición, lo que indica que es independiente de la posición $x / M$. 

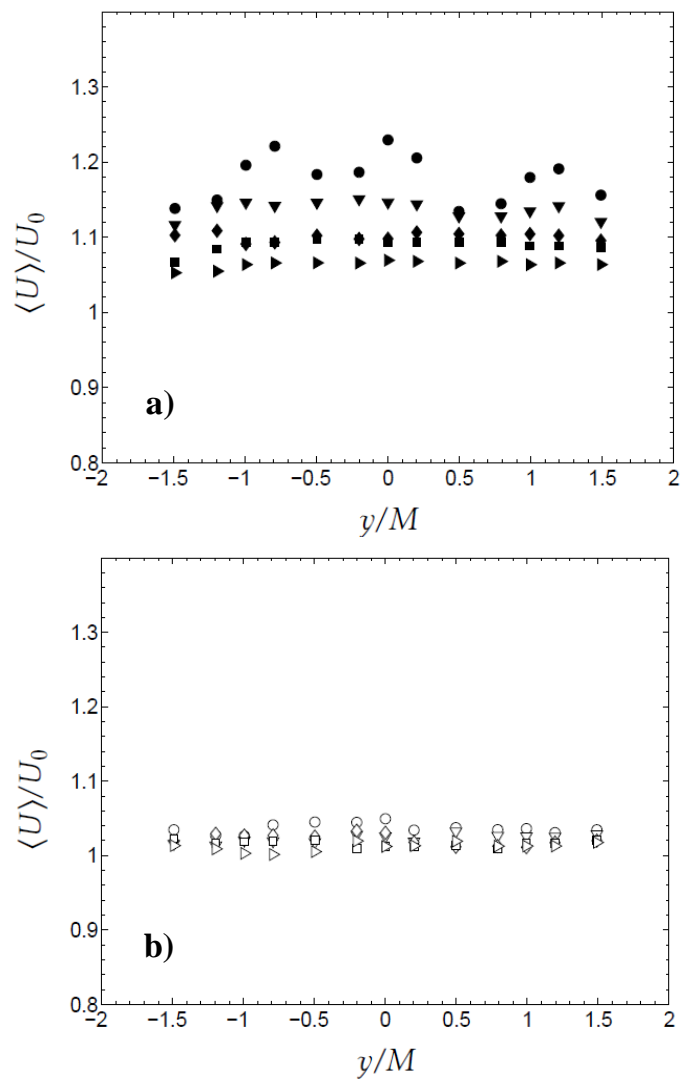

Figura 4. Distribución transversal de $\langle\mathrm{U}\rangle / U_{0}$ como función de $y / M$ para a) malla pasiva con $U_{0}=12 \mathrm{~m} / \mathrm{s}$ y

b) malla activa con $U_{0}=9 \mathrm{~m} / \mathrm{s}$. Los símbolos para la

malla pasiva son: $\bullet, \mathrm{x} / \mathrm{M}=4,75 ; \boldsymbol{\nabla}, \mathrm{x} / \mathrm{M}=9,75 ; \diamond, \mathrm{x} / \mathrm{M}$

$=14,75 ; \boldsymbol{\square}, \mathrm{x} / \mathrm{M}=19,75 ; \boldsymbol{\mathrm { x }} / \mathrm{M}=44,75 \mathrm{y}$ los

símbolos par la malla activa son: $\circ, \mathrm{x} / \mathrm{M}=29,18 ; \nabla$, $\mathrm{x} / \mathrm{M}=33,38 ; \diamond, \mathrm{x} / \mathrm{M}=37,58 ; \square, \mathrm{x} / \mathrm{M}=41,77 ;>, \mathrm{x} / \mathrm{M}=$ 45,97. Fuente: elaboración propia.

Con el objetivo de perfeccionar la estimación del inicio de la región homogénea e isotrópica, se usa la evolución longitudinal de la asimetría y la curtosis. La asimetría o skewness está definida como $S(u)=\left\langle u^{3}\right\rangle /\left(\left\langle u^{2}\right\rangle\right)^{3 / 2}$ y es una medida de la falta de simetría estadística en el flujo; la curtosis o flatness está definida como $K(u)=$ $\left\langle u^{4}\right\rangle /\left(\left\langle u^{2}\right\rangle\right)^{2}$ y es la medida de concentración de los datos alrededor del valor medio. En turbulencia homogénea e isotrópica las tres componentes de las fluctuaciones de la velocidad tienen una distribución de densidad de probabilidad muy cercana a una función normal; una distribución de probabilidad normal o Gaussiana tendrá un valor de asimetría de cero y una curtosis de 3.0 [13] [14].
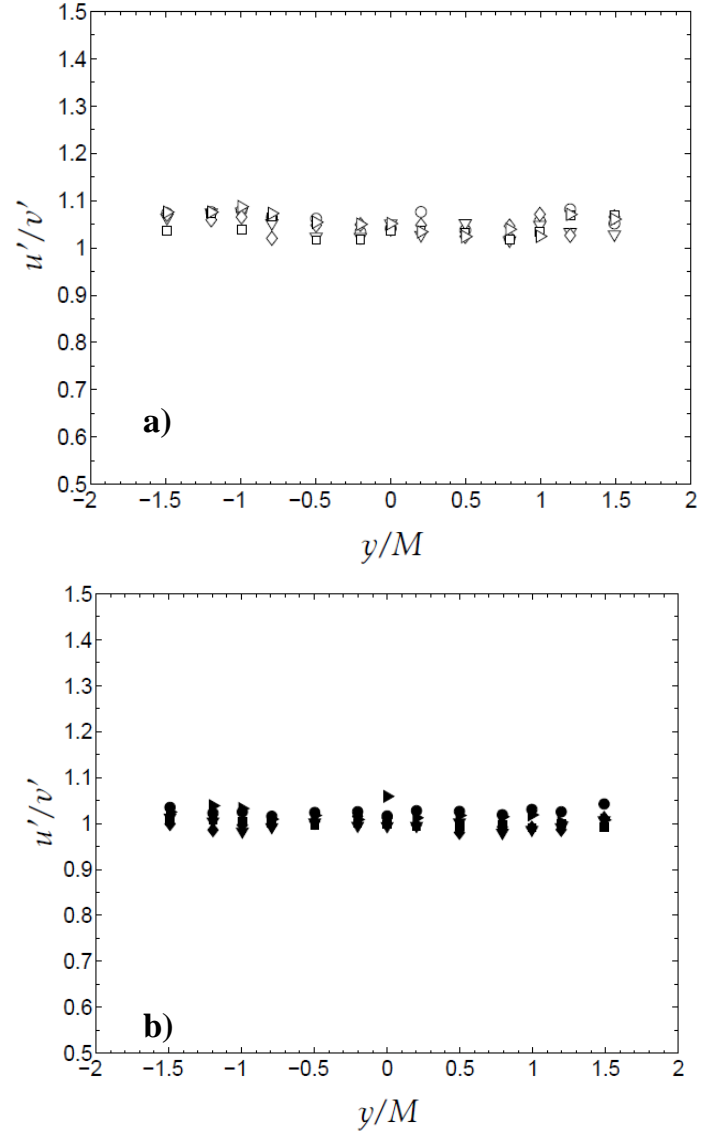

Figura 5. Factor de isotropía como función de y/M para a) malla pasiva con $U_{0}=12 \mathrm{~m} / \mathrm{s}$ y b) malla activa con $U_{0}=9 \mathrm{~m} / \mathrm{s}$. Los símbolos para la malla pasiva son: $\bullet$, $\mathrm{x} / \mathrm{M}=4,75 ; \boldsymbol{\nabla}, \mathrm{x} / \mathrm{M}=9,75 ; \diamond, \mathrm{x} / \mathrm{M}=14,75 ; \boldsymbol{\square}, \mathrm{x} / \mathrm{M}=$

19,$75 ; \mathrm{x} / \mathrm{M}=44,75$ y los símbolos par la malla activa son: $\circ, \mathrm{x} / \mathrm{M}=29,18 ; \nabla, \mathrm{x} / \mathrm{M}=33,38 ; \diamond, \mathrm{x} / \mathrm{M}=$

37,58; $\square, \mathrm{x} / \mathrm{M}=41,77 ;>, \mathrm{x} / \mathrm{M}=45,97$. Fuente: elaboración propia.

La asimetría puede tomar valores positivos y negativos, pero, de acuerdo con los experimentos observados en turbulencia, la asimetría debe ser negativa [13] [14], porque representa un proceso de transferencia de energía. La figura 6 muestra la evolución longitudinal de la asimetría y la curtosis de la velocidad longitudinal en el centro del túnel $(y / M=0)$.

El flujo se vuelve aproximadamente Gaussiano alrededor de $x / M=20$ para la malla pasiva donde $S(u) \approx 0,05 \mathrm{y}$ $K(u) \approx 2,95$. Estas estadísticas toman valores más altos para la malla activa, pero también se vuelven constantes alrededor de $x / M=30$, donde $S(u) \approx 0,3$ y $K(u) \approx 3,2$. 

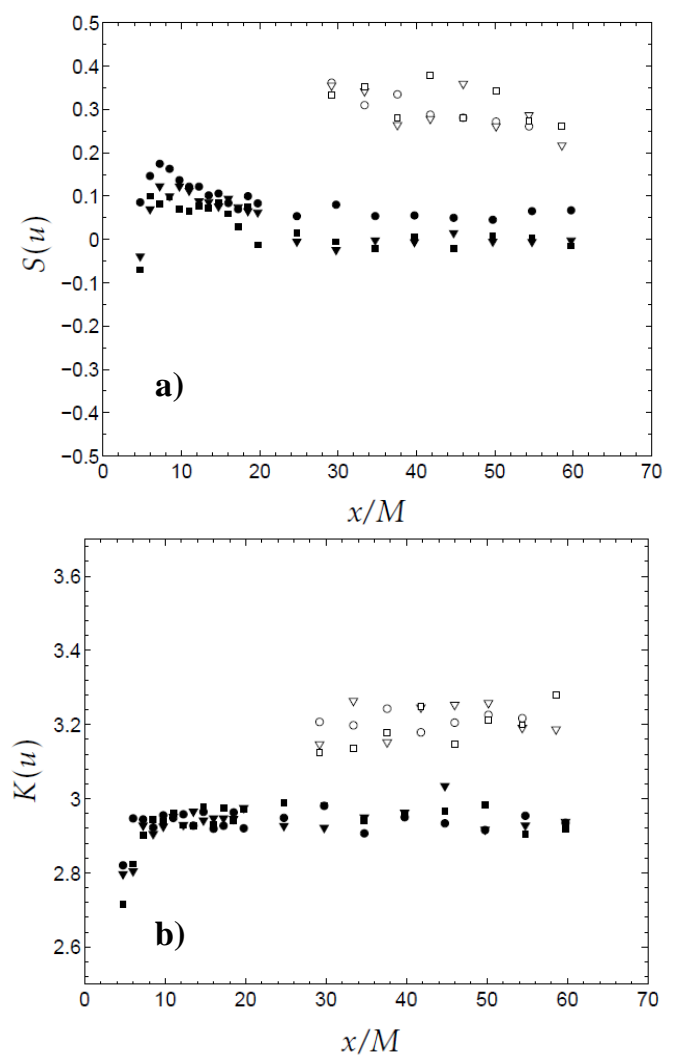

Figura 6. Evolución longitudinal de: a) la asimetría para $u$ y b) la curtosis para $u$. Los símbolos son: $\bullet$, malla pasiva $U_{0}=3 \mathrm{~m} / \mathrm{s} ; \boldsymbol{\nabla}$, malla pasiva $U_{0}=6 \mathrm{~m} / \mathrm{s} ; \mathbf{-}$, malla pasiva $U_{0}=12 \mathrm{~m} / \mathrm{s} ; \circ$, malla activa $U_{0}=3 \mathrm{~m} / \mathrm{s}$; $\nabla$, malla activa $U_{0}=6 \mathrm{~m} / \mathrm{s} ; \square$, malla activa $U_{0}=9$ $\mathrm{m} / \mathrm{s}$. Fuente: elaboración propia.

Estos resultados para la asimetría y la curtosis están en buen acuerdo con los estudios anteriores de [9] y [7]. Las gráficas para las variaciones de $S(v)$ y $K(v)$ no son mostradas, pero su comportamiento fue consistente con los de $S(u)$ y $K(u)$.

La figura 7 muestra la distribución de probabilidad (o PDF, por sus siglas en inglés Probability Density Function) de la velocidad longitudinal $u$, medida para una posición en el centro del túnel para las seis configuraciones o velocidades estudiadas en $x / M=44,75$ en la malla pasiva y $x / M=45,97$ en la malla activa, normalizada por la raíz de la media cuadrática, $\operatorname{PDF}\left[(u) / \sqrt{\left\langle u^{2}\right\rangle}\right]$, y han sido denotados como PDF (z).
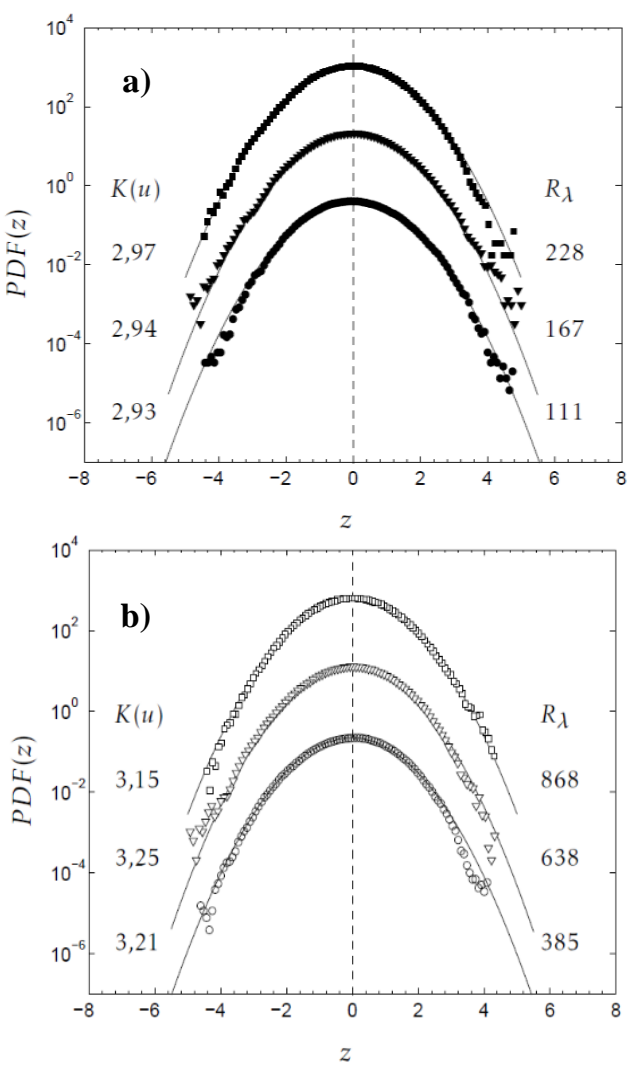

Figura 7. a) $P D F(z)$ para malla pasiva en $x / M=44,75 y$

b) PDF (z) para malla activa en $x / M=45,97$. Los símbolos son: $\bullet$, malla pasiva $U_{0}=3 \mathrm{~m} / \mathrm{s} ; \boldsymbol{\nabla}$, malla pasiva $U_{0}=6 \mathrm{~m} / \mathrm{s} ; \mathbf{m}$, malla pasiva $U_{0}=12 \mathrm{~m} / \mathrm{s} ; \circ$, malla activa $U_{0}=3 \mathrm{~m} / \mathrm{s} ; \nabla$, malla activa $U_{0}=6 \mathrm{~m} / \mathrm{s} ; \square$, malla activa $U_{0}=9 \mathrm{~m} / \mathrm{s}$. En la gráfica se han etiquetado la curtosis (a la izquierda) y $R_{\lambda}$ (a la derecha) para cada curva. Cada gráfica ha sido desplazada dos décadas con respecto a la curva más baja. Las líneas sólidas son

Gaussianas. Fuente: elaboración propia.

Las distribuciones de probabilidad son claramente cercanas a la distribución Gaussiana, debido a que los valores asimetría y curtosis son muy cercanos a cero y tres, respectivamente.

De acuerdo con los perfiles de velocidad media, del factor de isotropía, de la asimetría y la curtosis, el flujo fue considerado razonablemente homogéneo e isotrópico para posiciones $x / M>19,75$ en malla pasiva y $x /$ $M>29,18$ en malla activa. La tabla 1 muestra los principales parámetros del flujo para diferentes posiciones $x / M$ y diferentes velocidades $U_{0}$ para ambos tipos de malla. 


\subsection{Funciones de estructura}

\subsubsection{Exponentes de escala}

Una vez que se comprobó que el flujo fuera homogéneo e isotrópico, se procedió con el cálculo de los exponentes de las funciones de estructura. Los exponentes de escala fueron calculados usando el método ESS (por sus siglas en inglés Extended Self Similarity), propuesto por Benzi et al. [15], que permite el cálculo de los exponentes graficando la función de estructura de tercer orden en la abscisa frente a la función de estructura de cualquier otro orden en la ordenada. Benzi et al. [15] formularon lo siguiente:

$$
\Delta \boldsymbol{u}(\boldsymbol{x})^{n} \sim\left[\Delta \boldsymbol{u}(\boldsymbol{x})^{3}\right]^{\xi_{u}(x)}
$$

La convergencia estadística de la función de estructura de orden impar es significativamente más lenta que el de las órdenes pares. Para superar esto, han sustituido las funciones de estructura de la ecuación 3 por su módulo:

$$
\Delta \boldsymbol{u}(\boldsymbol{x})^{n} \sim\left[\left|\Delta \boldsymbol{u}(\boldsymbol{x})^{3}\right|\right]^{\xi_{u(x)}^{n}}
$$

Y establecieron además lo siguiente:

$$
\xi_{u(x)}^{n}=\frac{d\left|\Delta u(x)^{n}\right|}{d\left|\Delta u(x)^{3}\right|}
$$

La ecuación 5 también es válida para el cálculo de los exponentes transversales.

Si se representa $\xi_{u(x)}^{n}$ como función de $r / \eta$, existe una zona plana que indica la existencia de un exponente constante. En la figura 8 se han representado los valores de $\xi_{u(x)}^{n}$ para tres diferentes números de Reynolds y para valores de $n$ entre 2 y 8 ; se ha delimitado el inicio y el fin de esta zona plana con líneas punteadas verticales; las líneas horizontales punteadas corresponden al valor promedio del exponente $\xi_{u(x)}^{n}$ en esta zona delimitada. La determinación de los exponentes para órdenes más grandes que 6 es difícil para números de Reynolds altos, porque, por lo general, no es apreciable una zona plana donde el exponente sea constante, ese fenómeno indica que las funciones de estructura de alto orden son dominadas por eventos inusuales, que provocan que algunas estadísticas no converjan de manera satisfactoria [16].
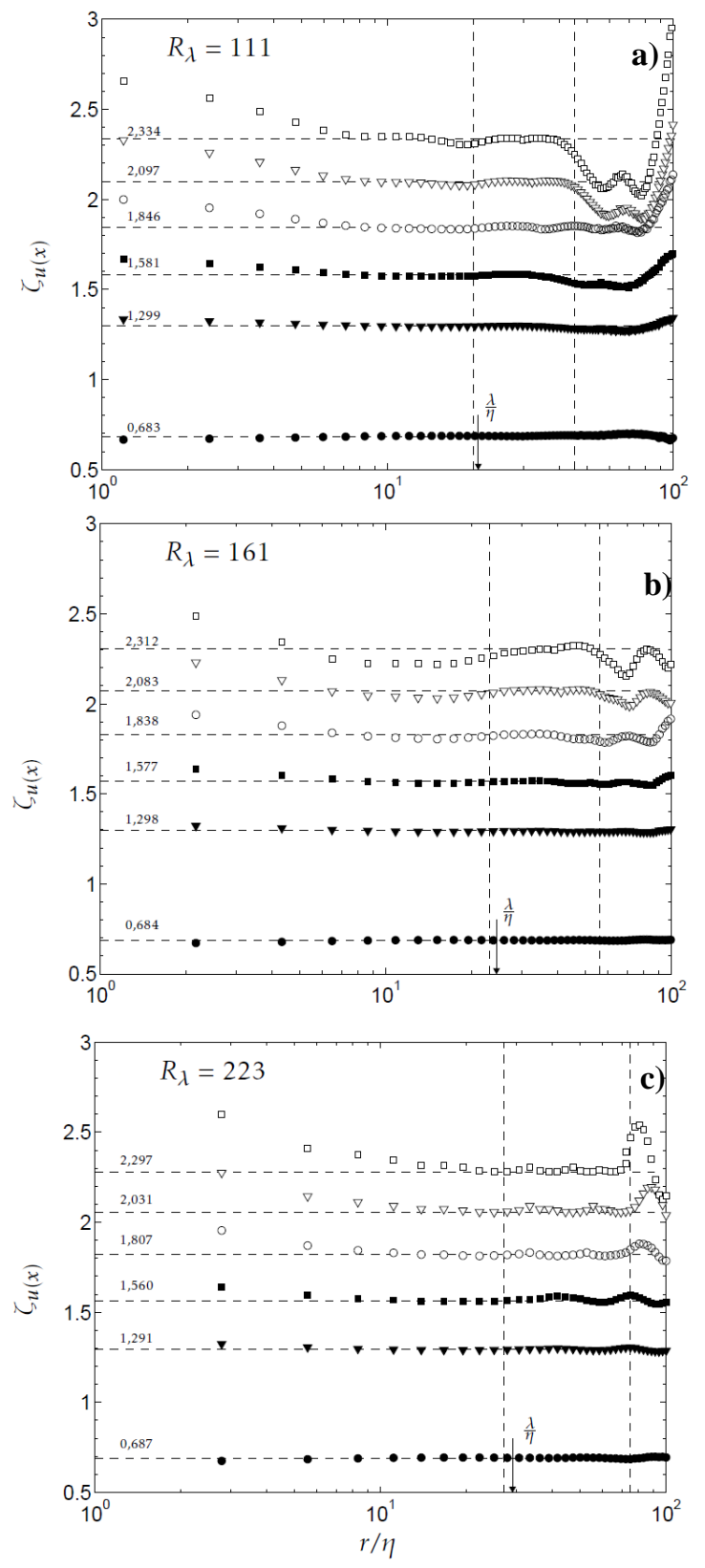

Figura 8. Exponentes longitudinales $\xi_{u(x)}^{n}$ evaluados usando el método ESS para a) Malla pasiva $U_{0}==3$ $\mathrm{m} / \mathrm{s}$ para $\mathrm{x} / \mathrm{M}=44,75$, b) Malla pasiva $U_{0}=6 \mathrm{~m} / \mathrm{s}$ para $\mathrm{x} / \mathrm{M}=19,75 \mathrm{y} \mathrm{c}$ ) Malla pasiva $U_{0}==12 \mathrm{~m} / \mathrm{s}$ para $\mathrm{x} / \mathrm{M}$

$=19,75$. Las líneas punteadas verticales indican la

región del rango inercial usada para evaluar los exponentes y las líneas horizontales corresponden a los valores $\operatorname{de} \xi_{u(x)}^{n}$ en este rango. Los símbolos son: •, orden $2 ; \boldsymbol{\nabla}$, orden $4 ; \boldsymbol{m}$, orden $5 ; \circ$, orden $6 ; \nabla$, orden 7 $\mathrm{y} \square$, orden 8 . Fuente: elaboración propia. 
Exponentes de escala de las funciones de estructura longitudinales y transversales en turbulencia homogénea e isotrópica generada en un túnel de viento

Tabla 2. Exponentes de escalamiento $\xi_{u(x)}^{n}$ y $\xi_{v(x)}^{n}$ obtenidos mediante el método ESS.

\begin{tabular}{|c|c|c|c|c|c|c|c|c|c|c|c|c|}
\hline & \multicolumn{6}{|c|}{ Malla pasiva } & \multicolumn{6}{|c|}{ Malla activa } \\
\hline$x / M$ & \multicolumn{6}{|c|}{44,75} & \multicolumn{6}{|c|}{45,97} \\
\hline $\boldsymbol{U}_{\mathbf{0}}(\mathrm{m} / \mathrm{s})$ & \multicolumn{2}{|c|}{3} & \multicolumn{2}{|c|}{6} & \multicolumn{2}{|c|}{12} & \multicolumn{2}{|c|}{3} & \multicolumn{2}{|c|}{6} & \multicolumn{2}{|c|}{9} \\
\hline$n$ & $\xi_{\mathbf{u}(\mathbf{x})}^{\mathbf{n}}$ & $\xi_{\mathrm{v}(\mathrm{x})}^{\mathrm{n}}$ & $\xi_{\mathbf{u}(\mathbf{x})}^{\mathbf{n}}$ & $\xi_{\mathrm{v}(\mathrm{x})}^{\mathrm{n}}$ & $\xi_{\mathbf{u}(\mathbf{x})}^{\mathbf{n}}$ & $\xi_{\mathrm{v}(\mathrm{x})}^{\mathrm{n}}$ & $\xi_{\mathbf{u}(\mathbf{x})}^{\mathbf{n}}$ & $\xi_{\mathbf{v}(\mathbf{x})}^{\mathbf{n}}$ & $\xi_{\mathbf{u}(\mathbf{x})}^{\mathbf{n}}$ & $\xi_{v(x)}^{n}$ & $\xi_{\mathbf{u}(\mathbf{x})}^{\mathbf{n}}$ & $\xi_{\mathbf{v}(\mathbf{x})}^{\mathbf{n}}$ \\
\hline 2 & 0,683 & 0,702 & 0,685 & 0,702 & 0,686 & 0,702 & 0,690 & 0,7053 & 0,697 & 0,710 & 0,702 & 0,710 \\
\hline 4 & 1,299 & 1,297 & 1,295 & 1,261 & 1,293 & 1,267 & 1,285 & 1,262 & 1,274 & 1,251 & 1,266 & 1,248 \\
\hline 5 & 1,581 & 1,501 & 1,572 & 1,493 & 1,565 & 1,505 & 1,556 & 1,478 & 1,522 & 1,468 & 1,501 & 1,452 \\
\hline 6 & 1,846 & 1,709 & 1,831 & 1,700 & 1,819 & 1,716 & 1,800 & 1,686 & 1,748 & 1,654 & 1,709 & 1,630 \\
\hline 7 & 2,097 & 1,896 & 2,073 & 1,888 & 2,056 & 1,903 & 2,011 & 1,852 & 1,955 & 1,785 & 1,896 & 1,770 \\
\hline 8 & 2,334 & 2,064 & 2,303 & 2,059 & 2,278 & 2,069 & 2,247 & 1,971 & 2,148 & 1,885 & 2,064 & 1,865 \\
\hline
\end{tabular}

Fuente: elaboración propia.

Tabla 3. Exponentes de escalamiento $\xi_{u(y)}^{n}$ obtenidos mediante el método ESS. Los exponentes $\xi_{u(y)}^{n}$ solo fueron medidos en malla activa para $U_{0}=3 \mathrm{~m} / \mathrm{s}, U_{0}=6 \mathrm{~m} / \mathrm{s}$ y $U_{0}=9 \mathrm{~m} / \mathrm{s}$.

\begin{tabular}{c|c|c|c|c|c|c|c|c|c}
\cline { 2 - 11 } & \multicolumn{9}{c}{ Malla activa } \\
\hline $\mathrm{x} / \mathbf{M}$ & \multicolumn{3}{|c|}{$\mathbf{2 9 , 1 8}$} & \multicolumn{3}{c}{$\mathbf{3 7 , 5 2}$} & \multicolumn{3}{c}{$\mathbf{4 5 , 9 7}$} \\
\hline $\boldsymbol{U}_{\mathbf{0}}$ & $\mathbf{3}$ & $\mathbf{6}$ & $\mathbf{9}$ & $\mathbf{3}$ & $\mathbf{6}$ & $\mathbf{9}$ & $\mathbf{3}$ & $\mathbf{6}$ & $\mathbf{9}$ \\
\hline $\boldsymbol{R}_{\boldsymbol{\lambda}}$ & 418 & 649 & 1118 & 352 & 637 & 1096 & 325 & 648 & 1066 \\
\hline$n$ & $\xi_{u(y)}^{n}$ & $\xi_{u(y)}^{n}$ & $\xi_{u(y)}^{n}$ & $\xi_{u(y)}^{n}$ & $\xi_{u(y)}^{n}$ & $\xi_{u(y)}^{n}$ & $\xi_{u(y)}^{n}$ & $\xi_{u(y)}^{n}$ & $\xi_{u(y)}^{n}$ \\
\hline 2 & 0,701 & 0,703 & 0,702 & 0,702 & 0,702 & 0,704 & 0,700 & 0,705 & 0,706 \\
4 & 1,263 & 1,260 & 1,263 & 1,264 & 1,262 & 1,258 & 1,267 & 1,257 & 1,254 \\
5 & 1,493 & 1,481 & 1,492 & 1,499 & 1,487 & 1,482 & 1,504 & 1,477 & 1,469 \\
6 & 1,690 & 1,669 & 1,686 & 1,710 & 1,679 & 1,672 & 1,713 & 1,669 & 1,649 \\
7 & 1,860 & 1,830 & 1,849 & 1,902 & 1,823 & 1,834 & 1,899 & 1,830 & 1,799 \\
8 & 2,007 & 1,970 & 1,987 & 2,056 & 1,945 & 1,977 & 2,068 & 1,969 & 1,924 \\
\hline
\end{tabular}

Fuente: elaboración propia.

Las tablas 2 y 3 muestran los resultados obtenidos mediante el método ESS para $\xi_{u(x)}^{n}, \xi_{v(x)}^{n}$ y $\xi_{u(y)}^{n}$, para diferentes posiciones $x / M$ y diferentes velocidades $U_{0}$ para ambos tipos de malla. Los exponentes $\xi_{u(y)}^{n}$ solo fueron medidos para la configuración de malla activa. En las tablas no se incluye ningún valor para los exponentes de escala de orden 3, porque para la metodología ESS el exponente para este orden es igual a 1,0.

Los valores obtenidos para $\xi_{u(x)}^{n}, \xi_{v(x)}^{n}$ y $\xi_{u(y)}^{n}$ son consistentes con los resultados de Toshiyuki et al. [16] y Shen et al. [17], quienes también utilizaron la metodología ESS para determinar los exponentes de escalamiento de las funciones de estructura longitudinales y transversales en sus experimentos.

La figura 9 muestra los exponentes de escalamiento $\xi_{u(x)}^{n}, \xi_{v(x)}^{n} \mathrm{y} \xi_{u(y)}^{n}$ graficados en función del orden $\mathrm{n}$, junto con los exponentes de escala de la teoría K41. Los símbolos $\bullet, \boldsymbol{\nabla}$ y representan los exponentes de escala para la malla pasiva, mientras los símbolos $\circ, \nabla \quad$ y $\square$ representan los exponentes para la malla activa.
Se observa claramente que el alejamiento respecto a la línea K41 es variable con el orden n considerado, cuanto más aumenta el orden, más se aleja el exponente de escalamiento de la predicción de Kolmogorov y es aún mayor la diferencia con respecto a K41 para el exponente de escalamiento transversal $\xi_{v(x)}^{n}$, que toma valores significativamente menores que los exponentes $\xi_{u(x)}^{n}$ y $\xi_{u(y)}^{n}$. Los exponentes de escala orden 2 y 4 parecen ser independientes del número de Reynolds y del tipo de malla, porque los valores obtenidos para estos órdenes no tienen diferencias significativas apreciables en los seis experimentos reportados; todos los puntos de las gráficas para estos ordenes convergen en el mismo valor. A medida que $n$ aumenta, se comienzan a notar diferencias cada vez más significativas entre exponentes; para $n>5$ se evidencia una clara dependencia de los exponentes de escala del tipo de malla y del número de Reynolds. Para la malla activa, los exponentes de escala, longitudinales y transversales, alcanzan valores más pequeños que los hallados para la malla pasiva. 

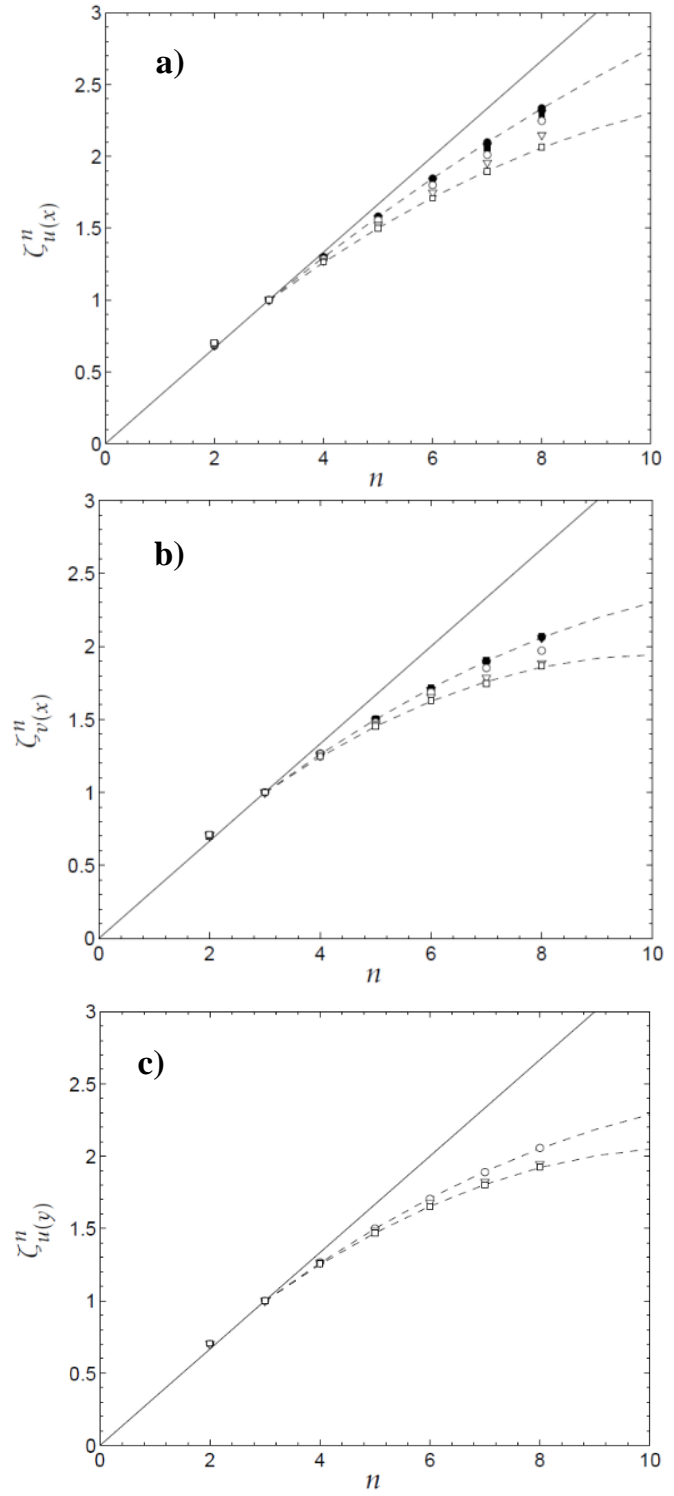

Figura 9. Exponentes de escalamiento: a) $\xi_{\mathrm{u}(\mathrm{x})}^{\mathrm{n}}$, b) $\xi_{\mathrm{v}(\mathrm{x})}^{\mathrm{n}}$ y c) $\xi_{\mathrm{u}(\mathrm{y})}^{\mathrm{n}}$ en función del orden n. Los símbolos son: $\bullet$, malla pasiva $U_{0}=3 \mathrm{~m} / \mathrm{s}$ con $R_{\lambda}=111$ en $x / M=44,75$;

$\boldsymbol{\nabla}$, malla pasiva $U_{0}=6 \mathrm{~m} / \mathrm{s}$ con $R_{\lambda}=167$ en $x / M=$

44,75; $\mathbf{n}$, malla pasiva $U_{0}=12 \mathrm{~m} / \mathrm{s}$ con $R_{\lambda}=228$ en $x / M$ $=44,75 ; \circ$, malla activa $U_{0}=3 \mathrm{~m} / \mathrm{s}$ con $R_{\lambda}=385 \mathrm{en}$ $x / M=45,97 ; \nabla$, malla activa $U_{0}=6 \mathrm{~m} / \mathrm{s}$ con $R_{\lambda}=638$ en $x / M=45,97$; $\square$, malla activa $U_{0}=9 \mathrm{~m} / \mathrm{s}$ con $R_{\lambda}=$

868 en $x / M=45,97$. La línea sólida representa la predicción K41 y las líneas punteadas son las líneas de tendencia para los exponente de escalamiento correspondientes al menor y mayor número de Reynolds. Fuente: elaboración propia.
En las figura 10 se han graficado los exponentes $\xi_{u(x)}^{n}$, $\xi_{v(x)}^{n}$ y $\xi_{u(y)}^{n}$ de orden 4, 6 y 8 , en función de $R_{\lambda}$ mientras se mantiene constante el grado $n$ de la función de estructura, para observar más detalladamente la variación de los exponente con el número de Reynolds y con el orden.

Los exponentes de escala longitudinales y transversales resultaron ser mayores que la predicción de Kolmogorov para la función de estructura de orden 2 en todas las velocidades estudiadas; para los órdenes de 4 a $8, \xi_{u(x)}^{n}$, $\xi_{v(x)}^{n} \mathrm{y} \quad \xi_{u(y)}^{n}$ fueron menores a K41, siempre permaneciendo el exponente de escala $\xi_{v(x)}^{n}$ aún más alejado de la predicción. $\xi_{u(y)}^{n}$ no mostró una fuerte dependencia de $R_{\lambda}$; razón por la cual los resultados no fueron graficados.

\section{Conclusiones}

Se ha estudiado el movimiento turbulento en turbulencia generada por una malla pasiva y una malla activa en un túnel de viento. La turbulencia fue estudiada para un intervalo de números de Reynolds basados en la escala de Taylor entre 100 y 1200. En general, la discusión se enmarcó en el contexto del estudio de los perfiles de velocidad, la evolución del factor de isotropía, asimetría y curtosis, las distribuciones de probabilidad y las funciones de estructura y sus exponentes de escala. Se demostró que para posiciones cercanas a la malla, $x / M<19,75$, el flujo medio normalizado se caracteriza por una pobre homogeneidad transversal. La falta de homogeneidad cercana a la malla comenzó a disminuir conforme aumentaba la distancia $\mathrm{x} / \mathrm{M}$.

De acuerdo con los perfiles de velocidad media, del factor de isotropía, de la asimetría y la curtosis, el flujo fue considerado razonablemente homogéneo e isotrópico para posiciones $x / M>19,75$ en malla pasiva y $x /$ $M>29,18$ en malla activa. La malla activa, debido a su relación de bloqueo constante al paso del aire, generó los números de Reynolds más altos.

Los resultados indicaron que los exponentes de escala de las funciones de estructura siguiente la metodología EES varían en función de $\mathrm{R}_{\lambda}$; cuanto más aumentaba $\mathrm{R}_{\lambda}$, mayor era la diferencia entre los exponentes de las funciones de estructura y los exponentes predichos por Kolmogorov en su teoría. Los exponentes de escala orden 2 y 4 fueron independientes del número de Reynolds y del tipo de malla, porque los valores obtenidos para estos órdenes no tienen diferencias significativas apreciables en los experimentos reportados. 

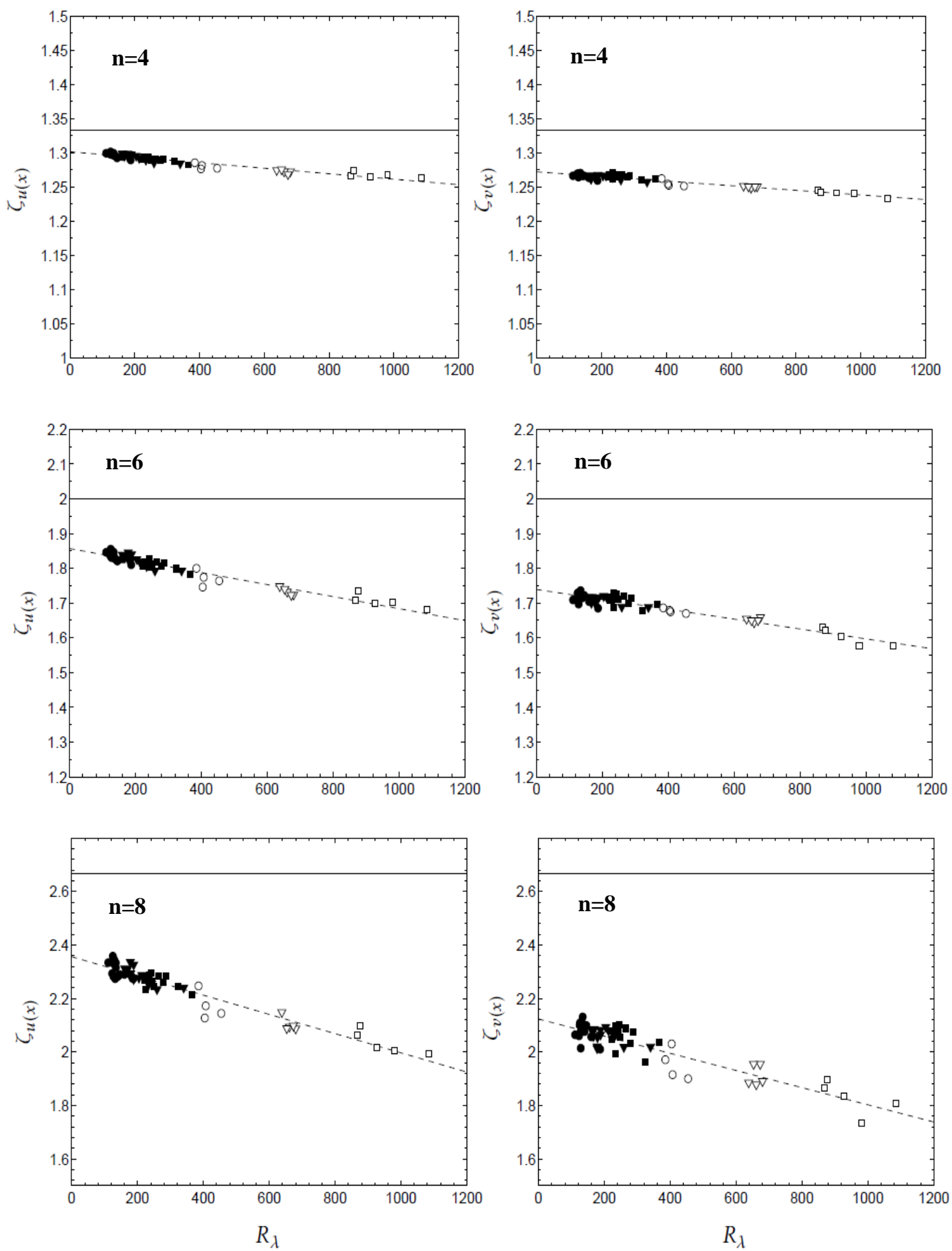

Figura 10. Exponentes de escala $\xi_{u(x)}^{n}$ y $\xi_{v(x)}^{n}$ de orden 4,6 y 8 en función de $R_{\lambda}$. Los símbolos son: $\bullet$, malla pasiva $U_{0}=3 \mathrm{~m} / \mathrm{s} ; \nabla$, malla pasiva $U_{0}=6 \mathrm{~m} / \mathrm{s} ; \mathbf{m}$, malla pasiva $U_{0}=12 \mathrm{~m} / \mathrm{s} ; \circ$, malla activa $U_{0}=3 \mathrm{~m} / \mathrm{s} ; \nabla$, malla activa $U_{0}=6 \mathrm{~m} / \mathrm{s} ; \square$, malla activa $U_{0}=9 \mathrm{~m} / \mathrm{s}$. La línea sólida representa la predicción de Kolmogorov y las líneas punteadas definen las líneas de tendencia para los exponentes de escala. Fuente: elaboración propia. 
Las líneas de tendencia de la figura 10 indicaron que $\xi_{\mathrm{u}(\mathrm{x})}^{\mathrm{n}}$ tiene una fuerte dependencia del número de Reynolds, pues los valores obtenidos para los exponentes varían significativamente entre el número de Reynolds más alto y más bajo. Se espera que cuando $\mathrm{R}_{\lambda} \rightarrow \infty$, el exponente $\zeta_{\mathrm{u}(\mathrm{x})}^{\mathrm{n}}$ sea el exponente que más aleje de la predicción K41. Estos resultados sugieren entonces que la postulación de Kolmogorov en su teoría K41 para los exponentes de escala es incorrecta; se propone entonces la realización de más experimentos con número de Reynolds más altos y con configuraciones de malla diferentes para validar o rechazar esta hipótesis.

\section{Agradecimientos}

El autor está agradecido con Zellman Warhaft, por su acompañamiento y guía para la realización de la fase experimental; gracias también a Juan Camilo Isaza, quien, como asesor, compartió su conocimiento y experiencia para llevar cabo esta investigación. Además, quiere dar las gracias a la Facultad de Ingeniería Mecánica y Aeroespacial de la Universidad de Cornell, por proveer los recursos e instalaciones necesarias para llevar a cabo cada uno de los experimentos; a la Universidad EAFIT, por su constante interés y apoyo en el desarrollo de esta investigación, y a la Universidad de Antioquia, por la motivación para el desarrollo de este estudio.

\section{Referencias}

[1] A. N. Kolmogorov, "Dissipation of Energy in Locally Isotropic Turbulence," Akad. Nauk SSSR Dokl., vol. 32, p. $16+, 1941$.

[2] H. Makita and K. Sassa, "Active Turbulence Generation in a Laboratory Wind Tunnel," in Advances in Turbulence 3, 1991, pp. 497-505. doi: 10.1007/978-3642-84399-0_54

[3] J. C. Isaza, R. Salazar, and Z. Warhaft, "On gridgenerated turbulence in the near- and far field regions," Journal of Fluid Mechanics, vol. 753, pp. 402426, 2014. doi: 10.1017/jfm.2014.375

[4] K. Yoon and Z. Warhaft, "The evolution of gridgenerated turbulence under conditions of stable thermal stratification," J. Fluid Mech., vol. 215, pp. 601-638, 1990. doi: 10.1017/S0022112090002786

[5] L. Mydlarski and Z. Warhaft, "On the onset of highReynolds-number grid-generated wind tunnel turbulence," J. Fluid Mech., vol. 320, pp. 331-368, 1996. doi: $10.1017 / \mathrm{S} 0022112096007562$
[6] H. H. Bruun, Hot wire anemometry: principles and signal analysis. Oxford University Press, 1995.

[7] P.-Å. KROGSTAD and P. A. DAVIDSON, "Freely decaying, homogeneous turbulence generated by multiscale grids," J. Fluid Mech., vol. 680, pp. 417-434, 2011. doi: 10.1017/jfm.2011.169

[8] A. Sirivat and Z. Warhaft, "The effect of a passive cross-stream temperature gradient on the evolution of temperature variance and heat flux in grid turbulence," $J$. Fluid Mech., vol. 128, no. 1, p. 323, Mar. 1983. doi: $10.1017 / \mathrm{S} 0022112083000506$

[9] P.-Å. KROGSTAD and P. A. DAVIDSON, "Is grid turbulence Saffman turbulence?," J. Fluid Mech., vol. 642, pp. 373-394, 2010. doi: $10.1017 / \mathrm{S} 0022112009991807$

[10] R. J. Hearst and P. Lavoie, "Decay of turbulence generated by a square-fractal-element grid," J. Fluid Mech., vol. 741, pp. 567-584, 2014. doi: $10.1017 / \mathrm{jfm} .2013 .684$

[11] Ö. ERTUNÇ, N. ÖZYILMAZ, H. LIENHART, F. DURST, and K. BERONOV, "Homogeneity of turbulence generated by static-grid structures," J. Fluid Mech., vol. 654, pp. 473-500, 2010. doi: $10.1017 / \mathrm{S} 0022112010000479$

[12] P. C. Valente and J. C. Vassilicos, "The decay of turbulence generated by a class of multiscale grids," $J$. Fluid Mech., vol. 687, pp. 300-340, 2011. doi: 10.1017/jfm.2011.353

[13] A. A. Townsend and G. K. Batchelor, "Decay of isotropic turbulence in the initial period," Proc. R. Soc. London A Math. Phys. Eng. Sci., vol. 193, no. 1035, pp. 539-558, 1948. doi: 10.1098/rspa.1948.0061

[14] L. F. G. Simmons and C. Salter, "Experimental investigation and analysis of the velocity variations in turbulent flow," Proc. R. Soc. London A Math. Phys. Eng. Sci., vol. 145, no. 854, pp. 212-234, 1934. doi: 10.1098/rspa.1934.0091

[15] R. Benzi, S. Ciliberto, R. Tripiccione, C. Baudet, F. Massaioli, and S. Succi, "Extended self-similarity in turbulent flows," Phys. Rev. E, vol. 48, no. 1, pp. R29-R32, Jul. 1993. doi: 10.1103/PhysRevE.48.R29

[16] T. Gotoh, D. Fukayama, and T. Nakano, "Velocity field statistics in homogeneous steady turbulence obtained using a high-resolution direct numerical 
Exponentes de escala de las funciones de estructura longitudinales y transversales en turbulencia homogénea

simulation," Phys. Fluids, vol. 14, no. 3, pp. 1065-1081, 2002. doi: $10.1063 / 1.1448296$

[17] X. Shen and Z. Warhaft, "Longitudinal and transverse structure functions in sheared and unsheared wind-tunnel turbulence," Phys. Fluids - PHYS FLUIDS, vol. 14, pp. 370-381, 2002. doi: 10.1063/1.1421059 